\title{
A new generalization of Aradhana distribution: Properties and applications
}

\author{
A. HASSAN, S. A. DAR, P. B. AHMAD AND B. A. PARA
}

\begin{abstract}
In this paper, we introduce a new generalization of Aradhana distribution called as Weighted Aradhana Distribution (WID). The statistical properties of this distribution are derived and the model parameters are estimated by maximum likelihood estimation. Simulation study of ML estimates of the parameters is carried out in R software. Finally, an application to real data set is presented to examine the significance of newly introduced model.
\end{abstract}

Mathematics Subject Classification 2010: 62E15, 60E05.

Keywords: Aradhana Distribution, Weighting Technique, Structural Properties and Maximum Likelihood Estimation, Simulation.

\section{INTRODUCTION}

Aradhana distribution is a newly proposed lifetime model formulated by Rama Shanker (2016) for several engineering applications and calculated its various characteristics including stochastic ordering, moments, order statistics, Renyi entropy, Stress-Strength reliability and ML estimation.

Probability density function (pdf) of Aradhana Distribution (AD) is given by

$$
f(x ; \theta)=\frac{\theta^{3}}{\theta^{2}+2 \theta+2}(1+x)^{2} e^{-\theta x} x>0, \theta>0
$$

The corresponding cdf of (1.1) is given by

$$
F(x ; \theta)=1-\left[1+\frac{\theta x(\theta x+2 \theta+2)}{\theta^{2}+2 \theta+2}\right] e^{-\theta x}, x>0, \theta>0
$$

\section{WEIGHTED ARADHANA DISTRIBUTION (WAD)}

Often scientists cannot select sampling units in observational studies with equal probability. Well defined sampling frames often do not exist for human, wildlife, insect, plant, or fish populations. Recorded observations on individuals in these 
populations are biased and will not have the original distribution unless every observation is given an equal chance of being recorded. Weighted distribution theory gives a unified approach for modeling these biased data. The concept of weighted distributions can be traced to the study of the effect of methods of ascertainment upon estimation of frequencies by Fisher (1934). Rao (1965) pointed out that in many situations the recorded observations cannot be considered as a random sample from the original distribution due to one or the other reason. Various weighted distributions have been discussed in Blumenthal (1963), Patil and Rao (1977,1978), Para and Jan (2018), Mahfoud and Patil (1982), Gupta and Kirmani (1990) and Hassan, Wani and Para (2018) among others.

Assume $\mathrm{X}$ is a non negative random variable with probability density function (pdf) $f(x)$. Let $W(x)$ be the weight function which is a non negative function, then the probability density function of the weighted random variable $X_{w}$ is given by:

$$
f_{w}(x)=\frac{W(x) f(x)}{E(w(x))}, \quad x>0,
$$

where $w(x)$ be a non-negative weight function and $E(w(x))=\int w(x) f(x) d x<\infty$.

In this paper, we have considered the weight function as $w(x)=x^{c}$ to obtain the weighted Aradhana model. The probability density of weighted Aradhana distribution is given as:

$$
\begin{gathered}
f_{w}(x, c, \theta)=\frac{x^{c} f(x, \theta)}{E\left[x^{c}\right]}, \\
f_{w}(x, c, \theta)=\frac{x^{c} \theta^{(c+3)}(1+x)^{2} e^{-\theta x}}{c !\left(\theta^{2}+2 \theta(c+1)+(c+1)(c+2)\right)}, \quad x>0, \theta>0, c>0,
\end{gathered}
$$

$$
\text { where } E\left(x^{c}\right)=\frac{c !\left(\theta^{2}+2 \theta(c+1)+(c+1)(c+2)\right)}{\theta^{c}\left(\theta^{2}+2 \theta+2\right)} \text {. }
$$

The corresponding cdf of weighted Aradhana Distribution (AID) is obtained as

$$
F_{w}(x ; c, \theta)=\int_{0}^{x} f_{w}(x ; c, \theta) d x
$$




$$
=\int_{0}^{x} \frac{x^{c} \theta^{(c+3)}(1+x)^{2} e^{-\theta x}}{c !\left(\theta^{2}+2 \theta(c+1)+(c+1)(c+2)\right)} d x \quad \text {, put } \theta x=t
$$

$\theta d x=d t$,

as $x \rightarrow 0, t \rightarrow 0$ and $x \rightarrow x, t \rightarrow \theta x \quad$, after simplification

$F_{w}(x ; c, \theta)=\frac{\theta^{2}}{c !\left(\theta^{2}+2 \theta(c+1)+(c+1)(c+2)\right)}\left(\gamma(c+1, \theta x)+\frac{1}{\theta^{2}} \gamma(c+3, \theta x)+\frac{2}{\theta} \gamma(c+2, \theta x)\right)$,

$x>0, \theta>0, c>0$,

where $\theta$ and $c$ are positive parameters and $\gamma(s, x)=\int_{0}^{x} t^{s-1} e^{-t} d t$ is a lower incomplete gamma function.

The graphs of probability density function and cumulative distribution function are plotted for different values of parameters $\theta$ and $c$ given in fig. 1 and fig. 2 respectively.

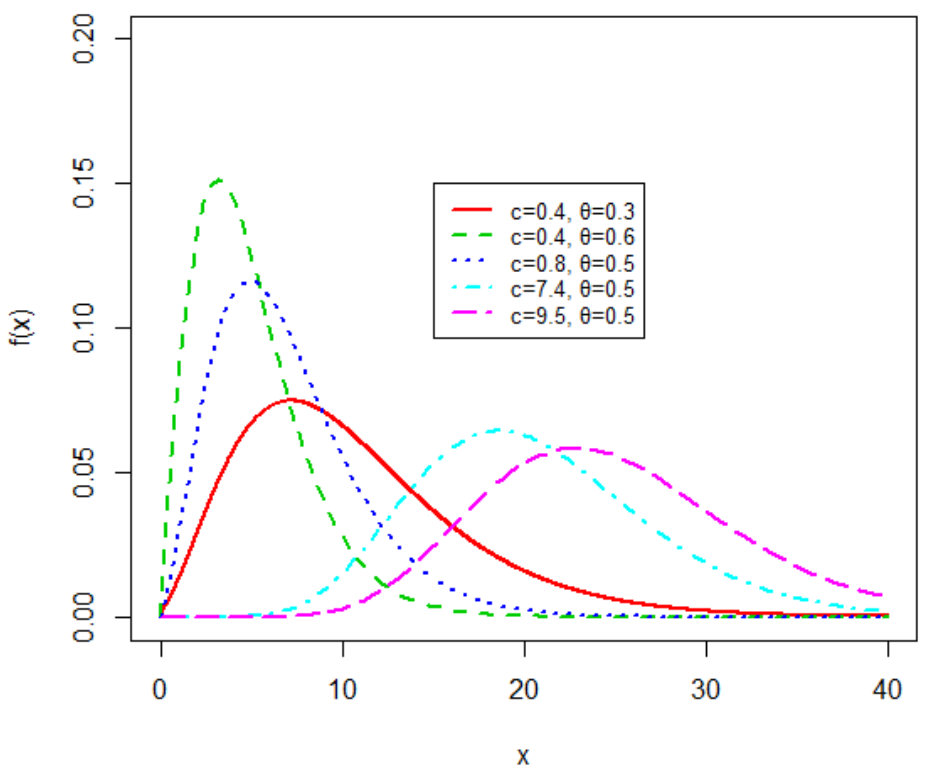

Fig. 1: Probability density function plot of Weighted Aradhana Distribution 
Fig. 1 gives the description of some of the possible shapes of weighed Aradhana distribution for different values of the parameters $\theta$ and $c$. It illustrates that the density function of weighted Aradhana distribution is positively skewed. For fixed $\theta$ it becomes more and more flatter as the value of $c$ is increased. Fig. 2 shows the graph of distribution function which is an increasing function.

\section{SPECIAL CASES}

Case 1: If we put $c=0$, then weighted Aradhana distribution (2.1) reduces to Aradhana distribution with probability density function as:

$f(x ; \theta)=\frac{\theta^{3}}{\theta^{2}+2 \theta+3}(1+x)^{2} e^{-\theta x} x>0, \theta>0$,

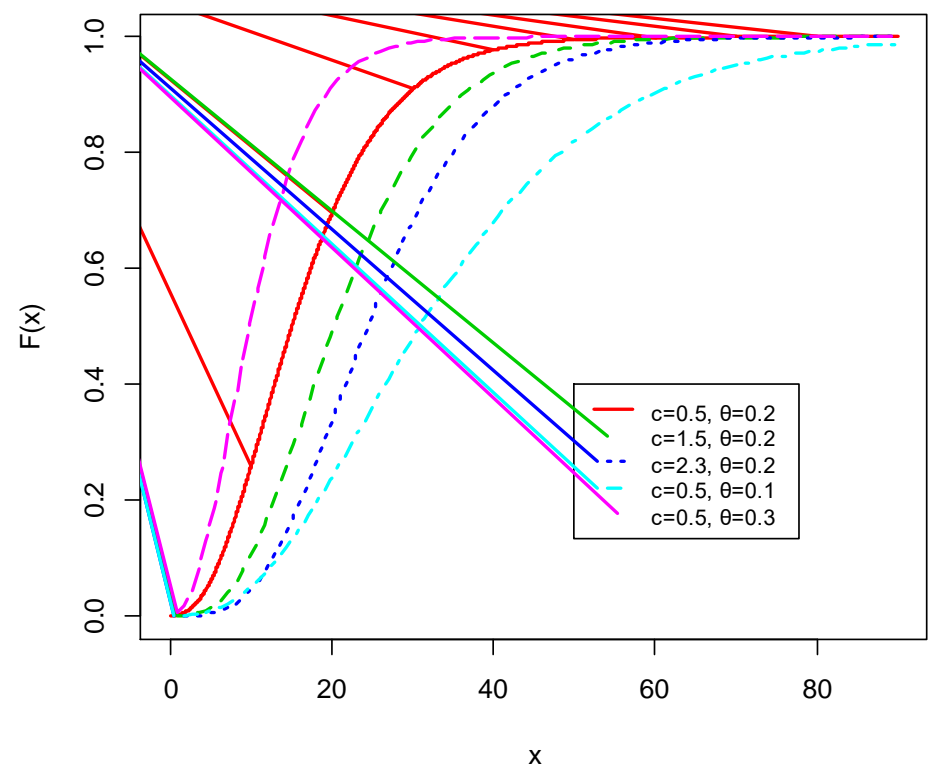

Fig.2: CDF plot of weighted Aradhana Distribution 


\section{RELIABILITY ANALYSIS}

In this section, we have obtained the reliability, hazard rate, reverse hazard rate of the proposed weighted Aradhana Distribution.

\subsection{Reliability function $R(x)$}

The reliability function is defined as the probability that a system survives beyond a specified time. It is also referred to as survival or survivor function of the distribution. It can be computed as complement of the cumulative distribution function of the model. The reliability function or the survival function of weighted Aradhana distribution is calculated as:

$$
\begin{gathered}
R_{w}(x, c, \theta)=1-\frac{\theta^{2}}{c !\left(\theta^{2}+2 \theta(c+1)+(c+1)(c+2)\right)}\left(\gamma(c+1, \theta x)+\frac{1}{\theta^{2}} \gamma(c+3, \theta x)+\frac{2}{\theta} \gamma(c+2, \theta x)\right) \\
x>0, \theta>0, c>0,
\end{gathered}
$$

The graphical representation of the reliability function for the weighted Aradhana distribution is shown in fig. 3.

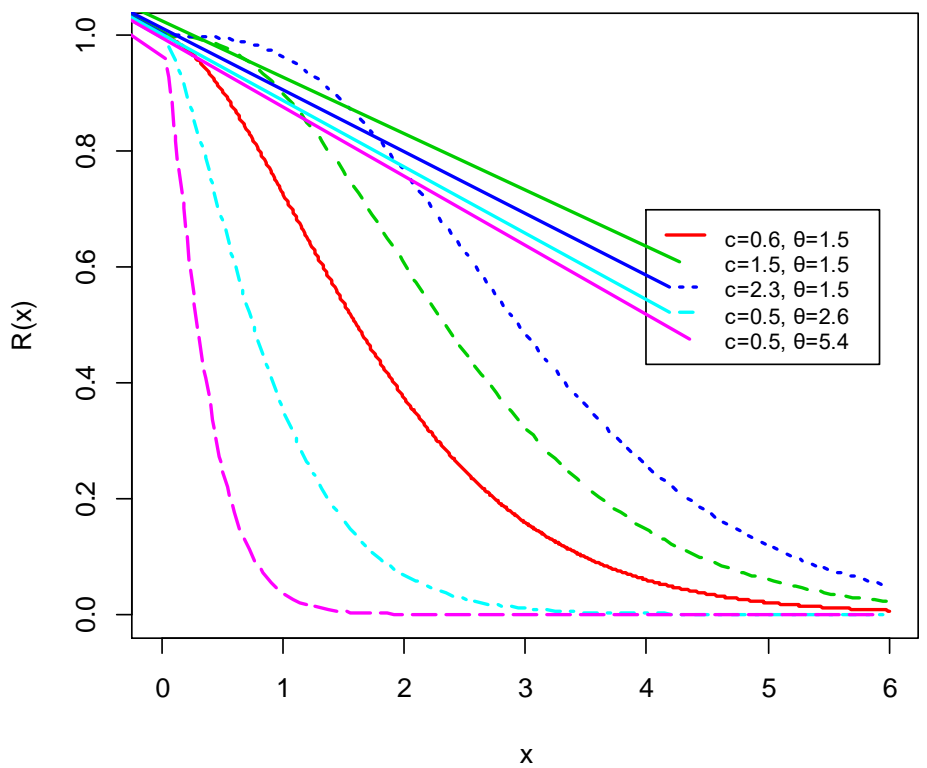

Fig. 3: Reliability function plot of weighted Aradhana Distribution 


\subsection{Hazard Function}

The hazard function is also known as hazard rate, instantaneous failure rate or force of mortality and is given as:

$$
\begin{gathered}
H . R=h(x ; c, \theta)=\frac{f_{w}(x, \theta)}{R_{w}(x, \theta)} . \\
h(x ; c, \theta)=\frac{x^{c} \theta^{c+3}(1+x)^{2} e^{-\theta x}}{c !\left(\theta^{2}+2 \theta(c+1)+(c+1)(c+2)\right)-\theta^{2}\left(\gamma(c+1, \theta x)+\frac{1}{\theta^{2}} \gamma(c+3, \theta x)+\frac{2}{\theta} \gamma(c+2, \theta x)\right)}
\end{gathered}
$$

\subsection{Reverse Hazard Rate}

The reverse hazard rate of the weighted Aradhana distribution are respectively given as:

$$
\begin{aligned}
& R . H . R=h_{r}(x, c, \theta)=\frac{f_{w}(x, \theta)}{F_{w}(x, \theta)}=\frac{x^{c} \theta^{c+3}(1+x)^{2} e^{-\theta x}}{\theta^{2}\left(\gamma(c+1, \theta x)+\frac{1}{\theta^{2}} \gamma(c+3, \theta x)+\frac{2}{\theta} \gamma(c+2, \theta, x)\right)}, \\
& x>0, \theta>0, c>0
\end{aligned}
$$

\section{STATISTICAL PROPERTIES}

In this section, the different structural properties of the proposed weighted Aradhana model have been evaluated. These include moments, harmonic mean, moment generating function and characteristic function

\subsection{Moments}

Suppose $\mathrm{X}$ is a random variable following weighted Aradhana distribution with parameter $\theta$, and then the rth moment for a given probability distribution is given by

$$
\begin{aligned}
\mu_{r}^{\prime}=E\left(X_{w}{ }^{r}\right) & =\int_{0}^{\infty} x^{r} f_{w}(x, c, \theta) d x \\
& =\int_{0}^{\infty} x^{r} \frac{x^{c} \theta^{(c+3)}(1+x)^{2} e^{-\theta x}}{c !\left(\theta^{2}+2 \theta(c+1)+(c+1)(c+2)\right)} d x
\end{aligned}
$$




$$
\begin{aligned}
& \mu_{r}^{\prime}=\frac{(c+r) !\left(\theta^{2}+(c+r+1)(c+r+2)+2 \theta(c+r+1)\right)}{\theta^{r} c !\left(\theta^{2}+2 \theta(c+1)+(c+1)(c+2)\right.} \\
& \mu_{1}^{\prime}=\frac{(c+1)\left(\theta^{2}+(c+2)(c+3)+2 \theta(c+2)\right)}{\theta\left(\theta^{2}+2 \theta(c+1)+(c+1)(c+2)\right)} \\
& V(X)=\frac{(c+1)(c+2)\left(\theta^{2}+(c+3)(c+4)+2 \theta(c+3)\right)}{\theta^{2}\left(\theta^{2}+2 \theta(c+1)+(c+1)(c+2)\right)}-\left[\frac{(c+1)\left(\theta^{2}+(c+2)(c+3)+2 \theta(c+2)\right.}{\theta\left(\theta^{2}+2 \theta(c+1)+(c+1)(c+2)\right)}\right]^{2}
\end{aligned}
$$

\subsection{Harmonic mean}

The harmonic mean for the proposed model is computed as:

$$
\begin{aligned}
H . M= & E\left[\frac{1}{X}\right]=\int_{0}^{\infty} \frac{1}{x} f_{w}(x ; c, \theta) d x \\
& =\int_{0}^{\infty} \frac{1}{x} \frac{x^{c} \theta^{c+3}(1+x)^{2} e^{-\theta x}}{c !\left(\theta^{2}+2 \theta(c+1)+(c+1)(c+2)\right)} d x \\
H . M & =\frac{c\left(\theta^{2}+2 \theta(c+1)+(c+1)(c+2)\right)}{\theta\left(\theta^{2}+c(c+1)+2 c \theta\right)}, \quad \theta>0, c>0,
\end{aligned}
$$

5.3. Moment generating function and Characteristic function of Weighted Aradhana Distribution (WAD)

We will derive moment generating function and characteristic function of WAD in this section.

THEOREM 1.1. If $X$ has the $\operatorname{WAD}(c, \theta)$, then the moment generating function $M_{X}(t)$ and the characteristic function $\psi_{X}(t)$ has the following form

$$
M_{X}(t)=\frac{\theta^{c+3}}{c !\left(\theta^{2}+2 \theta(c+1)+(c+1)(c+2)\right.}\left[\frac{(\theta-t)^{2}+(c+1)(c+2)+2(c+1)(\theta-t)}{(\theta-t)^{2}}\right]
$$

and $\psi_{X}(t)=\frac{\theta^{c+3}}{\theta^{2}+2 \theta(c+1)+(c+1)(c+2)}\left\{\frac{(\theta-i t)^{2}(c+1)(c+2)+2(c+1)(\theta-i t)}{(\theta-i t)^{2}}\right\}$

respectively. 
PROOF. We begin with the well known definition of the moment generating function given by

$$
\begin{aligned}
M_{X}(t) & =E\left(e^{t x}\right)=\int_{0}^{\infty} e^{t x} f(x ; \theta, c) d x \\
& =\frac{\theta^{c+3}}{c !\left(\theta^{2}+2 \theta(c+1)+(c+1)(c+2)\right.} \int_{0}^{\infty}\left[x^{c}\left(1+x^{2}+2 x\right) e^{-x(\theta-t)}\right] d x \\
& =\frac{\theta^{c+3}}{c !\left(\theta^{2}+2 \theta(c+1)+(c+1)(c+2)\right.} \int_{0}^{\infty}\left[\frac{c !}{(\theta-t)^{c+1}}+\frac{(c+2) !}{(\theta-t)^{c+3}}+\frac{2(c+1) !}{(\theta-t)^{c+2}}\right] \\
\Rightarrow M_{X}(t)= & \frac{\theta^{c+3}}{\left(\theta^{2}+2 \theta(c+1)+(c+1)(c+2)\right.}\left[\frac{(\theta-t)^{2}+(c+1)(c+2)+2(c+1)(\theta-t)}{(\theta-t)^{2}}\right]
\end{aligned}
$$

Also we know that $\psi_{X}(t)=M_{X}(i t)$

Therefore,

$$
\psi_{X}(t)=\frac{\theta^{c+3}}{\left(\theta^{2}+2 \theta(c+1)+(c+1)+(c+2)\right)}\left\{\frac{(\theta-i t)^{2}+(c+1)(c+2)+2(c+1)(\theta-i t)}{(\theta-i t)^{2}}\right\}
$$

\section{ORDER STATISTICS}

Let $X_{(1)}, X_{(2)}, X_{(3)} \ldots, X_{(n)}$ be the ordered statistics of the random sample $X_{1}, X_{2}, X_{3}, \ldots X_{n}$ drawn from the continuous distribution with cumulative distribution function $F_{X}(x)$ and probability density function $f_{X}(x)$, then the probability density function of rth order statistics $X_{(r)}$ is given by:

$$
f_{x(r)}(x, c, \theta)=\frac{n !}{(r-1) !(n-r) !} f(x)[F(x)]^{r-1}[1-F(x)]^{n-r} . \quad r=1,2,3, \ldots, n
$$

Using the equations (2.1) and (2.2), the probability density function of rth order statistics of weighted Aradhana distribution is given by: 


$$
\begin{aligned}
f_{w(r)}(x, c, \theta) & =\frac{n !}{(r-1) !(n-r) ! c !\left(\theta^{2}+2 \theta(c+1)+(c+1)(c+2)\right)} \\
& {\left[\frac{x^{c} \theta^{c+3}(1+x)^{2} e^{-\theta x}}{c !\left(\theta^{2}+2 \theta(c+1)+(c+1)(c+2)\right)}\left(\gamma(c+1, \theta x)+\frac{1}{\theta^{2}} \gamma(c+3, \theta x)+\frac{2}{\theta} \gamma(c+2, \theta x)\right)\right]^{r-1} } \\
& {\left[1-\frac{\theta^{2}}{c !\left(\theta^{2}+2 \theta(c+1)+(c+1)(c+2)\right)}\left(\gamma(c+1, \theta x)+\frac{1}{\theta^{2}} \gamma(c+3, \theta x)+\frac{2}{\theta} \gamma(c+2, \theta x)\right)\right]^{n-r} . }
\end{aligned}
$$

Then, the pdf of first order $X_{(1)}$ weighted Aradhana distribution is given by:

$$
\begin{aligned}
f_{w(1)}(x, c, \theta)= & n \frac{x^{c} \theta^{c+3}(1+x)^{2} e^{-\theta x}}{c !\left(\theta^{2}+2 \theta(c+1)+(c+1)(c+2)\right)} \\
& {\left[1-\frac{\theta^{2}}{c !\left(\theta^{2}+2 \theta(c+1)+(c+1)(c+2)\right)}\left(\gamma(c+1, \theta x)+\frac{1}{\theta^{2}} \gamma(c+3, \theta x)+\frac{2}{\theta} \gamma(c+2, \theta x)\right]^{n-1} .\right.}
\end{aligned}
$$

and the pdf of nth order $X_{(n)}$ weighted Aradhana model is given as:

$$
\begin{aligned}
f_{w(n)}(x, c, \theta)=n & \frac{x^{c} \theta^{c+3}(1+x)^{2} e^{-\theta x}}{c !\left(\theta^{2}+2 \theta(c+1)+(c+1)(c+2)\right)} \\
& {\left[\frac{\theta^{2}}{c !\left(\theta^{2}+2 \theta(c+1)+(c+1)(c+2)\right)}\left(\gamma(c+1, \theta x)+\frac{1}{\theta^{2}} \gamma(c+3, \theta x)+\frac{2}{\theta} \gamma(c+2, \theta x)\right)\right]^{n-1} . }
\end{aligned}
$$

\section{METHOD OF MAXIMUM LIKELIHOOD ESTIMATION OF WEIGHTED ARADHANA DISTRIBUTION}

This is one of the most useful method for estimating the different parameters of the distribution. Let $X_{1}, X_{2}, X_{3} \ldots . X_{n}$ be the random sample of size $\mathrm{n}$ drawn from weighted Aradhana distribution, then the likelihood function of weighted Aradhana distribution is given as:

$$
L(x \mid c, \theta)=\prod_{i=1}^{n} f(x ; c, \theta)=\prod_{i=1}^{n} \frac{x^{c} \theta^{c+3}(1+x)^{2} e^{-\theta x}}{c !\left(\theta^{2}+2 \theta(c+1)+(c+1)(c+2)\right)}
$$

The log likelihood function becomes:

$\log L=c \log \sum_{i=1}^{n} x_{i}+n(c+3) \log (\theta)+2 \log \sum_{i=1}^{n}\left(1+x_{i}\right)-\theta \sum_{i=1}^{n} x_{i}-n \log (c !)-n \log \left(\theta^{2}+2 \theta(c+1)+(c+1)(c+2)\right)$ 
Differentiating the log-likelihood function with respect to $\theta$ and $c$. This is done by partially differentiate (7.1) with respect to $\theta$ and $c$ and equating the result to zero, we obtain the following normal equations,

$\frac{d \log L}{d \theta}=\frac{n(c+3)}{\theta}-\sum_{i=1}^{n} x_{i}-\frac{n(2(\theta+c+1))}{\theta^{2}+2 \theta(c+1)+(c+1)(c+2)}=0$.

$\frac{d \log L}{d c}=\log \left(\sum_{i=1}^{n} x_{i}\right)+n \log \theta+\frac{n}{c !}-\frac{n(2 \theta+2 c+3)}{\log \left(\theta^{2}+2 \theta(c+1)+(c+1)(c+2)\right)}=0$

By solving equations (7.2) and (7.3), the maximum likelihood estimators of the parameters of the weighted Aradhana distribution are obtained using the numerical methods like Newton Raphson method.

We can compute the maximized unrestricted and restricted log likelihoods to construct the likelihood ratio (LR) statistics for testing the significance of weighted parameter of the proposed model. For example, we can use LR test to check whether the fitted weighted Aradhana distribution for a given data set is statistically "superior" to the fitted Aradhana distribution. In any case, hypothesis tests of the type $H_{0}: \Theta=\Theta_{0}$ versus $H_{1}: \Theta \neq \Theta_{0}$ can be performed using LR statistics. In this case, the LR statistic for testing $H_{0}$ versus $H_{1}$ is $\omega=2\left(L(\hat{\Theta})-L\left(\hat{\Theta}_{0}\right)\right)$ where $\hat{\Theta}$ and $\hat{\Theta}_{0}$ are the MLEs under $H_{1}$ and $H_{0}$. The statistic $\omega$ is asymptotically $($ as $n \rightarrow \infty)$ distributed as $\chi_{k}^{2}$, with $\mathrm{k}$ degrees of freedom which is equal to the difference in dimensionality of $\hat{\Theta}$ and $\hat{\Theta}_{0} . H_{0}$ will be rejected if the LR-test $\mathrm{p}$-value is $<0.05$ at $95 \%$ confidence level.

\subsection{Simulation Study of ML estimators of Weighted Aradhana Distribution}

Using R statistical software for simulation study of Maximum Likelihood (ML) estimates, we study the performance of ML estimators for different sample sizes $(\mathrm{n}=25,75,100,200,400)$. Using inverse CDF technique for data generation from WAD, the process was repeated 500 times for calculation of bias, variance and Mean Square Error (MSE). For six random parameter combinations of WAD, decreasing 
trend is being observed in average bias, variance and MSE as we increase the sample size (see table 1). Hence, performance of ML estimators is quite well and consistent in case of WAD.

Table I: Average bias, variance and MSE of ML estimates of WAD for different sample sizes

\begin{tabular}{|c|c|c|c|c|c|c|c|}
\hline \multirow[t]{2}{*}{ Parameter } & \multirow[t]{2}{*}{$\mathrm{n}$} & \multicolumn{3}{|c|}{$\mathrm{c}=0.5, \theta=0.3$} & \multicolumn{3}{|c|}{$\mathrm{c}=0.9, \theta=0.7$} \\
\hline & & Bias & Variance & MSE & Bias & Variance & MSE \\
\hline c & 25 & 0.316084 & 0.106875 & 0.206784 & 0.314656 & 0.398373 & 0.497381 \\
\hline$\theta$ & & 0.054112 & 0.004628 & 0.007556 & 0.14309 & 0.058252 & 0.078726 \\
\hline $\mathrm{c}$ & 75 & 0.072541 & 0.04108 & 0.046343 & 0.010907 & 0.063456 & 0.063575 \\
\hline$\theta$ & & 0.026196 & 0.002303 & 0.002989 & 0.036994 & 0.017707 & 0.019076 \\
\hline c & 100 & 0.082578 & 0.028625 & 0.035444 & 0.051299 & 0.040644 & 0.043275 \\
\hline$\theta$ & & 0.029879 & 0.002561 & 0.003454 & 0.047157 & 0.008902 & 0.011125 \\
\hline $\mathrm{c}$ & 200 & 0.067269 & 0.019194 & 0.023719 & 0.058119 & 0.026055 & 0.029433 \\
\hline$\theta$ & & 0.026875 & 0.002309 & 0.003031 & 0.064354 & 0.004743 & 0.008885 \\
\hline c & 400 & 0.039515 & 0.013038 & 0.014599 & 0.083429 & 0.020617 & 0.027578 \\
\hline$\theta$ & & 0.025358 & 0.002233 & 0.002876 & 0.029984 & 0.00469 & 0.005589 \\
\hline Parameter & $\mathrm{n}$ & \multicolumn{3}{|c|}{$\mathrm{c}=1.2, \theta=0.9$} & \multicolumn{3}{|c|}{$\mathrm{c}=1.8, \theta=1.5$} \\
\hline & & Bias & Variance & MSE & Bias & Variance & MSE \\
\hline c & 25 & 0.20579 & 0.293873 & 0.336222 & 0.312345 & 0.295641 & 0.393201 \\
\hline$\theta$ & & 0.181872 & 0.111975 & 0.145052 & 0.211940 & 0.181030 & 0.225949 \\
\hline $\mathrm{c}$ & 75 & 0.17945 & 0.15103 & 0.183232 & 0.203705 & 0.264116 & 0.305612 \\
\hline$\theta$ & & 0.112415 & 0.035996 & 0.048633 & 0.161424 & 0.108448 & 0.134506 \\
\hline c & 100 & 0.109011 & 0.088331 & 0.100214 & 0.151620 & 0.147000 & 0.169988 \\
\hline$\theta$ & & 0.084267 & 0.034166 & 0.041267 & 0.125316 & 0.049200 & 0.064904 \\
\hline $\mathrm{c}$ & 200 & 0.011496 & 0.020992 & 0.021125 & 0.089401 & 0.077759 & 0.085752 \\
\hline$\theta$ & & 0.017037 & 0.014832 & 0.015123 & 0.078205 & 0.044283 & 0.050398 \\
\hline c & 400 & 0.001589 & 0.016442 & 0.016445 & 0.082926 & 0.044597 & 0.051474 \\
\hline$\theta$ & & 0.019839 & 0.006001 & 0.006394 & 0.047921 & 0.022859 & 0.025155 \\
\hline
\end{tabular}




\begin{tabular}{|c|c|c|c|c|c|c|c|}
\hline \multirow[t]{2}{*}{ Parameter } & \multirow[t]{2}{*}{$\mathrm{n}$} & \multicolumn{3}{|c|}{$\mathrm{c}=2.5, \theta=1.7$} & \multicolumn{3}{|c|}{$\mathrm{c}=2.8, \theta=2.5$} \\
\hline & & Bias & Variance & MSE & Bias & Variance & MSE \\
\hline $\mathrm{c}$ & 25 & 0.342660 & 0.567742 & 0.685158 & 0.325616 & 1.161363 & 1.267389 \\
\hline$\theta$ & & 0.327808 & 0.383191 & 0.490649 & 0.295561 & 0.544709 & 0.632065 \\
\hline $\mathrm{c}$ & 75 & 0.131818 & 0.210193 & 0.227569 & 0.425697 & 0.452776 & 0.633994 \\
\hline$\theta$ & & 0.092191 & 0.111219 & 0.119718 & 0.328474 & 0.239748 & 0.347643 \\
\hline c & 100 & -0.028951 & 0.094912 & 0.095750 & 0.286518 & 0.190818 & 0.272910 \\
\hline$\theta$ & & 0.058024 & 0.046838 & 0.050205 & 0.259910 & 0.143187 & 0.210740 \\
\hline $\mathrm{c}$ & 200 & -0.023804 & 0.104879 & 0.105446 & 0.137308 & 0.125422 & 0.144275 \\
\hline$\theta$ & & 0.003426 & 0.073616 & 0.073628 & 0.101188 & 0.068870 & 0.079109 \\
\hline $\mathrm{c}$ & 400 & 0.042858 & 0.065325 & 0.067162 & 0.039300 & 0.068572 & 0.070116 \\
\hline$\theta$ & & 0.057663 & 0.028284 & 0.031608 & 0.030030 & 0.032064 & 0.032966 \\
\hline
\end{tabular}

\section{LIKELIHOOD RATIO TEST}

Let $x_{1}, x_{2}, \ldots, x_{n}$ be a random sample drawn from Aradhana distribution or weighted Aradhana distribution. We test the hypothesis $H_{0}: f(x)=f(x, \theta) v / s H_{1}: f(x)=f_{w}(x, \theta)$

In order to test whether the random sample come from Aradhana distribution or weighted Aradhana distribution, we use the following test statistic

$$
\begin{aligned}
& \Delta=\frac{L_{1}}{L_{0}}=\prod_{i=1}^{n} \frac{f_{w}(x, \theta)}{f(x, \theta)} \\
& \Delta=\frac{L_{1}}{L_{0}}=\prod_{i=1}^{n} \frac{\frac{x^{c} \theta^{c+3}(1+x)^{2} e^{-\theta x}}{c !\left(\theta^{2}+2 \theta(c+1)+(c+1)(c+2)\right)}}{\frac{\theta^{3}(1+x)^{2} e^{-\theta x}}{\theta^{2}+2 \theta+2}} \\
& \Delta=\frac{\theta^{c n}\left(\theta^{2}+2 \theta+2\right)^{n} \prod_{i=1}^{n} x_{i}^{c}}{(c !)^{n}\left(\theta^{2}+2 \theta(c+1)+(c+1)(c+2)\right)^{n}}
\end{aligned}
$$


We reject the null hypothesis if

$$
\begin{aligned}
& \Delta=\left[\frac{\theta^{c}\left(\theta^{2}+2 \theta+2\right)}{c !\left(\theta^{2}+2 \theta(c+1)+(c+1)(c+2)\right)}\right]^{n} \prod_{i=1}^{n} x_{i}^{c}>k \\
& \Delta^{*}=\prod_{i=1}^{n} x_{i}^{c}>k^{*} \quad \text { where }^{*}=k\left[\frac{c !\left(\theta^{2}+2 \theta(c+1)+(c+1)(c+2)\right)}{\theta^{c}\left(\theta^{2}+2 \theta+2\right)}\right]^{n}
\end{aligned}
$$

For a large sample of size $n, 2 \log \Delta$ is distributed as chi-squared distribution with one degree of freedom. Also we reject the null hypothesis when probability value is given by $p\left(\Delta^{*}>\beta^{*}\right)$, where $\beta^{*}=\prod_{i=1}^{n} x_{i}$ is less than a specified level of significance, where $\prod_{i=1}^{n} x_{i}$ is the observed value of the statistic $\Delta^{*}$.

\section{APPLICATIONS OF WEIGHTED ARADHANA DISTRIBUTIONS}

To illustrate the significance of the suggested model, a real life example is presented. The goodness of fit result of the suggested model (Weighted Aradhana Distribution) is compared with the base model (Aradhana Distribution). In this case, we analyze the strength data, which was originally reported by Badar and Priest (1982) and it represents the strength measured in GPA for single carbon fibers and impregnated 1000-carbon fiber tows. Single fibers were tested under tension at gauge lengths of $10 \mathrm{~mm}$ with sample sizes $\mathrm{n}=63$. Surles and Padgett (2001) also studied this data set. The data is given in table 2 .

Table II: Strength data set (gauge lengths of $10 \mathrm{~mm}$ ).

\begin{tabular}{|l|l|l|l|l|l|l|l|l|l|l|}
\hline 1.901 & 2.132 & 2.203 & 2.228 & 2.257 & 2.35 & 2.361 & 2.396 & 2.397 & 2.445 & 2.454 \\
\hline 2.474 & 2.518 & 2.522 & 2.525 & 2.532 & 2.575 & 2.614 & 2.616 & 2.618 & 2.624 & 2.659 \\
\hline 2.675 & 2.738 & 2.74 & 2.856 & 2.917 & 2.928 & 2.937 & 2.937 & 2.977 & 2.996 & 3.03 \\
\hline 3.125 & 3.139 & 3.145 & 3.22 & 3.223 & 3.235 & 3.243 & 3.264 & 3.272 & 3.294 & 3.332 \\
\hline 3.346 & 3.377 & 3.408 & 3.435 & 3.493 & 3.501 & 3.537 & 3.554 & 3.562 & 3.628 & 3.852 \\
\hline 3.871 & 3.886 & 3.971 & 4.024 & 4.027 & 4.225 & 4.395 & 5.02 & & & \\
\hline
\end{tabular}


By fitting Aradhana distribution and weighted Aradhana distribution to this data set, we observed that weighted Aradhana distribution fits statistically well as compared to Aradhana distribution. For comparison of the two distributions, we consider the criteria like AIC (Akaike information criterion), AICC (corrected Akaike information criterion) and BIC (Bayesian information criterion). The better distribution corresponds to lesser AIC, AICC and BIC values.

$$
\mathrm{AIC}=2 \mathrm{k}-2 \log L \quad \mathrm{AICC}=\mathrm{AIC}+\frac{2 k(k+1)}{n-k-1} \text { and } \mathrm{BIC}=\mathrm{k} \log n-2 \log L
$$

where $\mathrm{k}$ is the number of parameters in the statistical model, $\mathrm{n}$ is the sample size and $-\log L$ is the maximized value of the $\log$-likelihood function under the considered model. From Table 3, it has been observed that Weighted Aradhana distribution have the lesser AIC, AICC, $-\log L$ and BIC values as compared to Aradhana Distribution. Hence we can conclude that the Weighted Aradhana distribution leads to a better fit than the Aradhana distribution. Also likelihood ratio test reveals that weighted parameter c plays statistically significant role for data set given in table 2 .

Table III: ML estimates, AIC, AICC, BIC, - $\log$ L Criterion and Likelihood Ratio Test values for strength data set (gauge lengths of $10 \mathrm{~mm}$ ).

\begin{tabular}{|l|l|l|l|l|l|l|l|}
\hline Criteria & $\widehat{\boldsymbol{\theta}}$ & \multicolumn{1}{|c|}{$\widehat{\boldsymbol{c}}$} & $-\log \mathbf{L}$ & AIC & AICC & BIC & $\begin{array}{l}\text { Ratio } \\
\text { Statistic }\end{array}$ \\
\hline $\begin{array}{l}\text { Aradhana } \\
\text { Distribution }\end{array}$ & $\begin{array}{l}0.766 \\
(0.05)\end{array}$ & - & 112.075 & 226.150 & 226.216 & 228.293 & \multirow{2}{*}{110.36} \\
\hline $\begin{array}{l}\text { Weighted } \\
\text { Aradhana } \\
\text { Distribution }\end{array}$ & $\begin{array}{l}8.49 \\
(1.49)\end{array}$ & $\begin{array}{l}23.4 \\
(4.52)\end{array}$ & 56.894 & 117.787 & 117.987 & 122.074 & \\
\hline
\end{tabular}




\section{CONCLUSION}

In the present study, we have introduced a new generalization of the Aradhana distribution called as Weighted Aradhana distribution. The subject distribution is generated by using the weighting technique and taking the one parameter Aradhana distribution as the base distribution. Some mathematical properties along with reliability measures are discussed. Model is fitted to real life data for examining its significance.

\section{REFERENCES}

[1] Bader, M. G., \& Priest, A. M. (1982). Statistical aspects of fiber and bundle strength in hybrid composites, In; hayashi T, Kawata K. Umekawa S (Eds.), Progress in Science in Engineering Composites, ICCM-IV Tokyo, 1129 -1136.

[2] Blumenthal, S. (1963). Proportional sampling in life length studies. Technometrics, 9 (2), 205-218.

[3] Fisher, R.A. (1934). The effects of methods of ascertainment upon the estimation of frequencies, The Annals of Eugenics, 6, 13-25.

[4] Gupta, R.C., \& Kirmani, S.N.U.A. (1990). The role of weighted distributions in stochastic modeling. Comm. Statist. Theory Meth. 19 (9), 3147-3162.

[5] Hassan, A., Wani, S.A., \& Para, B. A. (2018). On three Parameter Weighted Quasi Lindley Distribution: Properties and Applications, International Journal of Scientific Research in Mathematical and Statistical Sciences, 5(5), 210-224.

[6] Mahfoud, M., \& Patil, G.P. (1982). On weighted distributions. In: Kallianpur, G., Krishnaiah, P.R., Ghosh, J.K. (Eds.), Statistics and Probability: Essays in Honor of C.R. Rao. North-Holland, Amsterdam, pp. 479-492.

[7] Para, B.A., \& Jan, T. R. (2018). On three Parameter Weighted Pareto Type II Distribution: Properties and Applications in Medical Sciences. Applied Mathematics and Information Sciences Letters, 6 (1), 13-26.

[8] Patil, G.P., Rao, C.R. \& Zelen, M. (1988). Weighted distributions, Encyclopedia of Statistical Sciences, 9, 565-571.

[9] Patil, G.P., Rao, C.R. (1977). In: Krishnaiah, P.R. (Ed.), The Weighted Distributions: A Survey and Their Applications. North-Holand, Amsterdam, pp. 383 -405.

[10] Patil, G.P., Rao, C.R. (1978). Weighted distributions and size-biased sampling with applications to wild-life populations and human families. Biometrics, 34, 179-189.

[11] Rao, C.R., (1965). On discrete distributions arising out of methods of ascertainment. In: Patil, G.P. (Ed.), Classical and Contagious Discrete Distributions. Permagon Press, Oxford and Statistical Publishing Society, Calcutta, pp. 320-332. 
[12] Surles, J.G., Padgett, W.J. (2001). Inference for reliability and stress-strength for a scaled Burr type X distribution. Lifetime Data Anal, 7, 187-200.

Anwar Hassan

Department of Statistics,

University of Kashmir, Srinagar, India.

Showkat Ahmad Dar

Department of Statistics,

University of Kashmir, Srinagar, India.

Peer Bilal Ahmad

Department of Statistics,

Islamic University of Science and technology, Awantipora, J\&K, India.

Bilal Ahmad Para

Department of Statistics,

Government Degree College Boys Anantnag, J\&K, India.

email corresponding author: darshowkat2429@gmail.com 